\title{
Inventário de Elateridae (Coleoptera) de Vila Dois Rios, Ilha Grande, Angra Dos Reis, Rio de Janeiro
}

\author{
Vinícius Amaral Corrêa ${ }^{1}$, Sônia Aparecida Casari ${ }^{2}$ \& José Ricardo Miras Mermudes ${ }^{1,3}$ \\ ${ }^{1}$ Laboratório de Entomologia, Departamento de Zoologia, Universidade Federal do Rio de Janeiro - UFRJ, \\ CP 68044, CEP 21941-971, Rio de Janeiro, RJ, Brasil \\ ${ }^{2}$ Museu de Zoologia, Universidade de São Paulo-USP, CP 42494, CEP 04218-970, São Paulo, SP, Brasil \\ ${ }^{3}$ Autor para correspondência: José Ricardo Miras Mermudes, e-mail: jrmermudes@gmail.com
}

CORREAA,V.A., CASARI, S.A. \& MERMUDES, J.R.M. Inventory of the Elateridae (Coleoptera) from Vila Dois Rios, (Ilha Grande, Angra dos Reis, RJ. Biota Neotrop., 11(4): http://www.biotaneotropica.org.br/v11n4/ en/abstract?inventory+bn03211042011

\begin{abstract}
Results of an inventory of Elateridae species from the Atlantic Forest in Vila Dois Rios (Ilha Grande, Angra dos Reis, RJ) are presented. Seven collecting trips were conducted between January and December of 2008, and insects were collected manually by beating the vegetation, and using light traps on the trails near CEADS (Center for Environmental Studies and Sustainable Development, State University of Rio de Janeiro)-5 m altitude, $23^{\circ} 11^{\prime} 05^{\prime \prime} \mathrm{S}$ and $44^{\circ} 11^{\prime} 27^{\prime \prime} \mathrm{W}$. A total of 212 specimens were collected in five subfamilies, representing the first record of 19 genera and 28 species from Ilha Grande. Elaterinae was the richest and most dominate subfamily, with Pomachilius terminatus was the most abundantly species. Agrypninae and Cardiophorinae corresponded to $19.34 \%$ of total abundance, the former with 11 species and the latter with four. Lissominae and Denticollinae were rare with only a single species.
\end{abstract}

Keywords: new records, Atlantic Forest, Brazil.

CORRÊA,V.A., CASARI, S.A. \& MERMUDES, J.R.M. Inventário de Elateridae (Coleoptera) de Vila Dois Rios, Ilha Grande, Angra Dos Reis, Rio de Janeiro. Biota Neotrop., 11(4): http://www.biotaneotropica.org. br/v11n4/pt/abstract?inventory+bn03211042011

Resumo: Os resultados do inventário das espécies de Elateridae da Mata Atlântica em Vila Dois Rios (Ilha Grande, Angra dos Reis, RJ) são apresentados. Sete coletas foram realizadas entre janeiro e dezembro de 2008 com guarda-chuva entomológico e armadilha luminosa em trilhas nas proximidades do Centro de Estudos Ambientais e Desenvolvimento Sustentável, da Universidade do Estado do Rio de Janeiro (CEADS), 5 m altitude, $23^{\circ} 11^{\prime} 05^{\prime}$ S e 44 11'27' O. No total foram capturados 212 espécimes para cinco subfamílias, representando o primeiro registro de 19 gêneros e 28 espécies para a Ilha Grande. Elaterinae foi a subfamília mais rica e dominante com Pomachilius terminatus destacando-se como a espécie mais abundante. Agrypninae e Cardiophorinae corresponderam a 19,34\% da abundância total, a primeira mais rica com 11 espécies e a segunda com quatro. As subfamílias raras foram Lissominaee e Denticollinae, com apenas um representante.

Palavras-chave: novos registros, Mata Atlântica, Brasil. 


\section{Introdução}

Os elaterídeos são reconhecidos por sua habilidade de saltar, fazendo ruído característico. Possuem uma combinação de caracteres que inclui corpo geralmente achatado, de forma alongada e estreita, protórax livremente articulado e de tamanho desproporcional comparado ao resto do corpo, pernas delgadas, antenas geralmente serradas, espinho prosternal bem desenvolvido com encaixe na fosseta mesosternal e tarsos pentâmeros. Geralmente apresentam cores pouco vistosas, de castanho a preto; muitos com manchas amareladas ou avermelhadas. A capacidade de saltar quando colocados sobre o dorso, produzindo um 'click' audível, resulta da forte pressão do encaixe do espinho prosternal na fosseta mesosternal. Também inclui espécies bioluminescentes. Os adultos são fitófagos, consumindo sucos de plantas e geralmente coletados na vegetação. As larvas podem ser saprófagas, fitófagas ou predadoras. Ingerem alimento líquido e apresentam digestão extra-oral. Algumas espécies principalmente dos gêneros Agriotes Eschscholtz, Athous Eschscholtz, Cardiophorus Eschscholtz, Ctenicera Latreille, Conoderus Eschscholtz e Melanotus Eschscholtz são consideradas pragas, atacando cereais e agricultura (Lima 1953; Lawrence \& Newton 1995; Johnson 2002; Costa et al. 2010).

Elateridae é a maior família da série Elateriformia e da Superfamília Elateroidea sendo a nona família mais diversa de coleópteros, com pelo menos 400 gêneros e quase 4.000 espécies. Está dividida em 17 subfamílias: Agrypninae, Campyloxeninae, Cardiophorinae, Cebrioninae, Denticollinae, Elaterinae, Eudicronychinae, Hemiopinae, Lissominae, Morostominae, Negastriinae, Oxynopterinae, Physodactylinae, Pityobiinae, Semiotinae, Subprotelaterinae e Thylacosterninae (Costa et al. 2010). No Brasil encontram-se em torno de 590 espécies, cerca de $7 \%$ da fauna global de Elateridae (Costa 2000).

Considerando que o Bioma Mata Atlântica está altamente degradado, com apenas $8 \%$ de sua cobertura vegetal original, e possui ainda acentuado número de espécies endêmicas (Mittermeier et al. 2005), nenhum registro de elaterídeos fora realizado, especificamente no Rio de Janeiro. Inventariar as espécies neste bioma torna-se importante, pois auxiliará com informações que permitam no futuro melhores estratégias de conservação.

\section{Material e Métodos}

As coletas foram realizadas em Vila Dois Rios, na Ilha Grande, Angra dos Reis, litoral sul do Estado do Rio de Janeiro. A caracterização da vegetação do local foi descrita em Rodrigues et al. (2010). De janeiro a dezembro de 2008 sete coletas foram realizadas em quatro trilhas de Vila Dois Rios : 1) trilha do Caxadaço; 2) trilha da Jararaca; 3) trilha da Parnaioca; e 4) Trilha do Cavalinho. As entradas das trilhas ficam próximas das coordenadas $23^{\circ} 11^{\prime} 05^{\prime \prime} \mathrm{S}$ e $44^{\circ} 11^{\prime} 27^{\prime \prime}$ O onde se localiza a base de pesquisa Centro de Estudos Ambientais e Desenvolvimento Sustentável, da Universidade do Estado do Rio de Janeiro (CEADS), altitude de $5 \mathrm{~m}$ no lado oceânico da ilha. Foram feitas captura por quatro pessoas com três guardachuvas entomológicos, totalizando 140 horas de coleta. Além disso, foi utilizada uma armadilha luminosa, com pano branco (18-24 horas).

A lista do material determinado é apresentada em ordem alfabética de subfamília, tribo, gênero, e espécies ou morfoespécies (sp.). A distribuição geográfica das espécies identificadas nos resultados tiveram como base o catálogo Blackwelder (1944) e artigos de citados no item distribuição, pois não existe no momento um inventário regional para as espécies de Elateridae.

Siglas utilizadas no texto: DZRJ, Coleção Entomológica Prof. José Alfredo Pinheiro Dutra, Departamento de Zoologia, Universidade Federal do Rio de Janeiro, Rio de Janeiro, Brasil; MNRJ, Museu Nacional, Universidade Federal do Rio de Janeiro, Rio de Janeiro,
Brasil; MZSP, Museu de Zoologia da Universidade de São Paulo, São Paulo, Brasil; UERJ, Coleção Entomológica do Laboratório de Entomologia do Departamento de Zoologia da Universidade do Estado do Rio de Janeiro, Rio de Janeiro, Brasil.

\section{Resultados e Discussão}

Neste inventário foram reconhecidas 5 subfamílias, 8 tribos, 19 gêneros e 28 espécies representadas por 212 espécimes coletados. Das espécies encontradas, uma foi identificada ao nível de tribo e 10 ao nível de gênero o que reflete a falta de conhecimento referente à fauna de elaterídeos da região. Elaterinae foi a mais abundante e diversa (Figura 1) correspondendo a 59,43\% do total capturado. Agrypninae e Cardiophorinae corresponderam a 19,34\% cada uma, enquanto Denticollinae e Lissominae representaram 1,42 e $0,47 \%$, respectivamente. A espécie mais abundante foi Pomachilius terminatus Candèze, 1860, com mais de 26,89\% da fauna coletada (Figura 2).

Abaixo segue a lista das espécies:

\section{SUBFAMÍLIA AGRYPNINAE}

TRIBO Agrypnini Candèze

\section{1-Dilobitarsus petiginosus Germar, 1840 (Figura 3)}

Distribuição (Blackwelder 1944): Brasil.

Material Examinado: BRASIL, Rio de Janeiro: Angra dos Reis (Ilha Grande, Vila Dois Rios) Trilha do Caxadaço, 1) 11.X.2008, Projeto Coleoptera col. (DZRJ).

\section{TRIBO Oophorini Gistel}

\section{2-Aeolus flavipennis Candéze, 1859 (Figura 4)}

Distribuição (Blackwelder 1944): Brasil.

Material Examinado: BRASIL, Rio de Janeiro: Angra dos Reis (Ilha Grande, Vila Dois Rios) Trilha do Cavalinho, 1) 12.X.2008, 1) 11.XII.2008; Trilha do Caxadaço, 1) 26.I.2008, 1) 07.IX.2008, 3, 10.X.2008, 3, 11.X.2008; Trilha da Jararaca, 2) 12.XII.2008; Trilha da Parnaioca, 1) 05.IX.2008, 1) 06.IX.2008, 1) 10.X.2008, 2) 11.X.2008, 2) 12.X.2008, 2) 13. XII.2008, Projeto Coleoptera col. (DZRJ, MZSP).

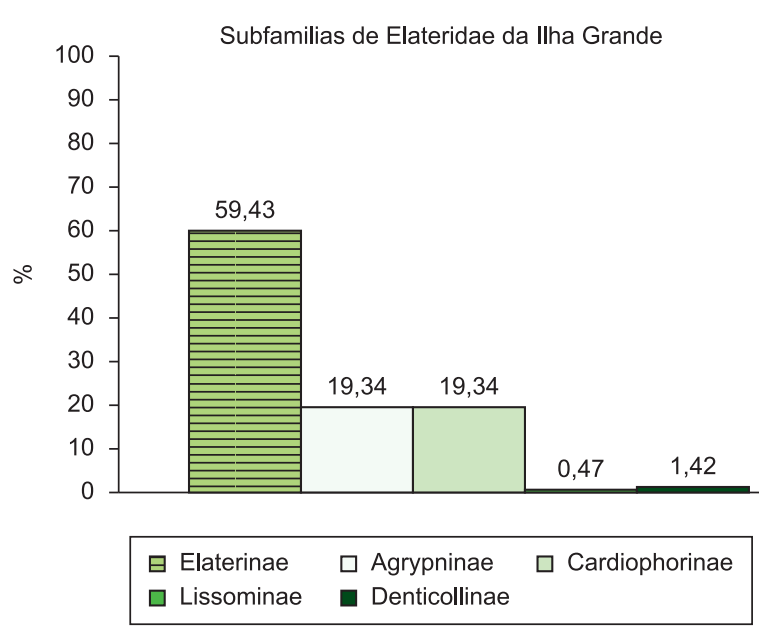

Figura 1. Abundância das subfamílias de Elateridae coletadas em Vila Dois Rios (Ilha Grande, Angra dos Reis, RJ, Brasil).

Figure 1. Abundance of the subfamilies of Elateridae collected in Vila Dois Rios (Ilha Grande, Angra dos Reis, RJ, Brazil). 


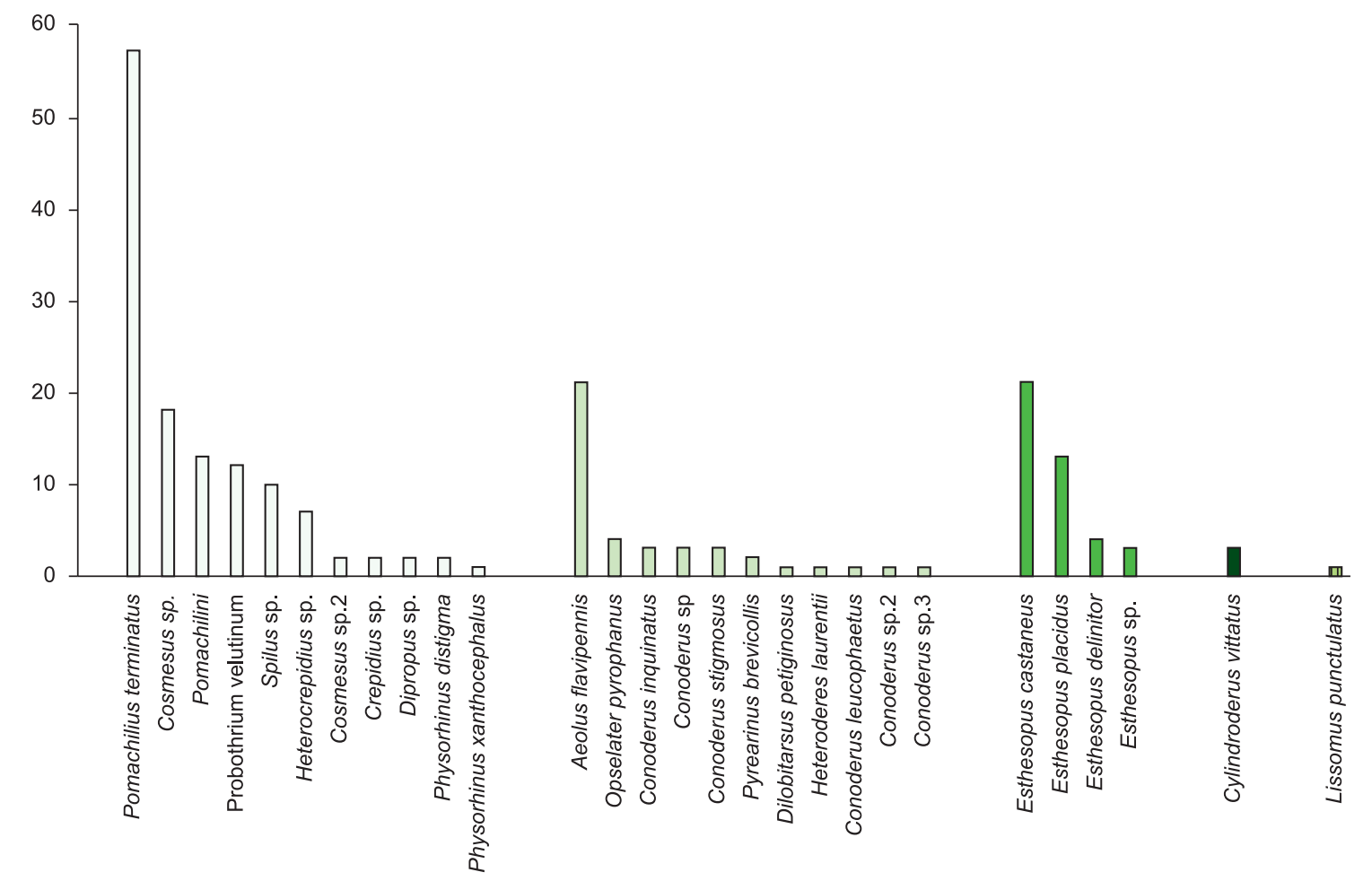

$\square$ Subfamília Elaterinae $\square$ Subfamília Agrypninae $\square$ Subfamília Cardiophorinae $\square$ Subfamília Denticollinae $\square$ Subfamília Lissominae

Figura 2. Abundância das espécies coletadas em Vila Dois Rios (Ilha Grande, Angra dos Reis, RJ, Brasil).

Figure 2. Abundance of species collected in Vila Dois Rios (Ilha Grande, Angra dos Reis, RJ, Brazil).

\section{3-Heteroderes laurentii (Guérin 1838) (Figura 5)}

Distribuição (Blackwelder, 1944): Antilhas (Porto Rico, St. Vincent, Mustique, Grenada), Brasil, Peru, Paraguai, Argentina.

Material Examinado: BRASIL, Rio de Janeiro: Angra dos Reis (Ilha Grande, Vila Dois Rios), Armadilha Luminosa, 1) 12.X.2008, Projeto Coleoptera col. (DZRJ).

4-Conoderus inquinatus (Candèze, 1859) (Figura 6)

Distribuição (Blackwelder 1944): Brasil.

Material Examinado: BRASIL, Rio de Janeiro: Angra dos Reis (Ilha Grande, Vila Dois Rios) Armadilha Luminosa,

1) 12.X.2008, Trilha do Caxadaço, 2) 16.III.2008, Projeto

Coleoptera col. (DZRJ, MZSP).

5-Conoderus leucophaetus (Candèze 1859) (Figura 7)

Distribuição (Candéze, 1859, Blackwelder, 1944): Brasil

(Bahia), Argentina.

Material Examinado: BRASIL, Rio de Janeiro: Angra dos Reis (Ilha Grande, Vila Dois Rios) Trilha da Parnaioca, 1) 25.I.2008, Projeto Coleoptera col. (DZRJ).

6-Conoderus stigmosus (Germar, 1839) (Figura 8)

Distribuição (Blackwelder 1944): Brasil.

Material Examinado: BRASIL, Rio de Janeiro: Angra dos

Reis (Ilha Grande, Vila Dois Rios) Trilha da Parnaioca, 2) 05. IX.2008, 1) 06.IX.2008, Projeto Coleoptera col. (DZRJ, MZSP).

7-Conoderus sp.1 (Figura 9)

Material Examinado: BRASIL, Rio de Janeiro: Angra dos Reis (Ilha Grande, Vila Dois Rios) Trilha da Parnaioca, 2) 05. IX.2008, 1) 10.X.2008, Projeto Coleoptera col. (DZRJ, MZSP).

\section{8-Conoderus sp.2 (Figura 10)}

Material examinado: BRASIL, Rio de Janeiro: Angra dos Reis (Ilha Grande, Vila Dois Rios) Trilha da Parnaioca, 1) 13. XII.2008, Projeto Coleoptera col. (DZRJ).

9-Conoderus sp.3 (Figura 11)

Material examinado: BRASIL, Rio de Janeiro: Angra dos Reis (Ilha Grande, Vila Dois Rios), 1) 14.IX.2008, Projeto Coleoptera col. (DZRJ).

\section{TRIBO PYROPHORINI}

10-Opselater pyrophanus (Illiger, 1807) (Figura 12)

Distribuição. De acordo com Rosa (2004) ocorre no Brasil (Goiás, Mato Grosso, Pernambuco, Alagoas, Bahia, Espírito Santo, Rio de Janeiro, Minas Gerais, São Paulo, Paraná, Santa Catarina, Rio Grande do Sul) e Argentina (Pico).

Material Examinado: BRASIL, Rio de Janeiro: Angra dos Reis (Ilha Grande, Vila Dois Rios) Trilha do Caxadaço, 2) 26.I.2008, 2) 25.I.2008. Projeto Coleoptera col. (DZRJ, MZSP).

11-Pyrearinus brevicollis (Eschscholtz, 1829) (Figura 13)

Distribuição (Costa 1979): Brasil (RJ).

Material Examinado: BRASIL, Rio de Janeiro: Angra dos Reis (Ilha Grande, Vila Dois Rios) Trilha do Caxadaço, 1) 26.I.2008; Trilha da Parnaioca, 1) 26.I.2008, Projeto Coleoptera col. (DZRJ).

\section{SUBFAMÍLIA CARDIOPHORINAE}

12-Esthesopus castaneus Esch, 1829 (Figura 14)

Distribuição (Candèze, 1960): Brasil (Rio de Janeiro e Bahia). 

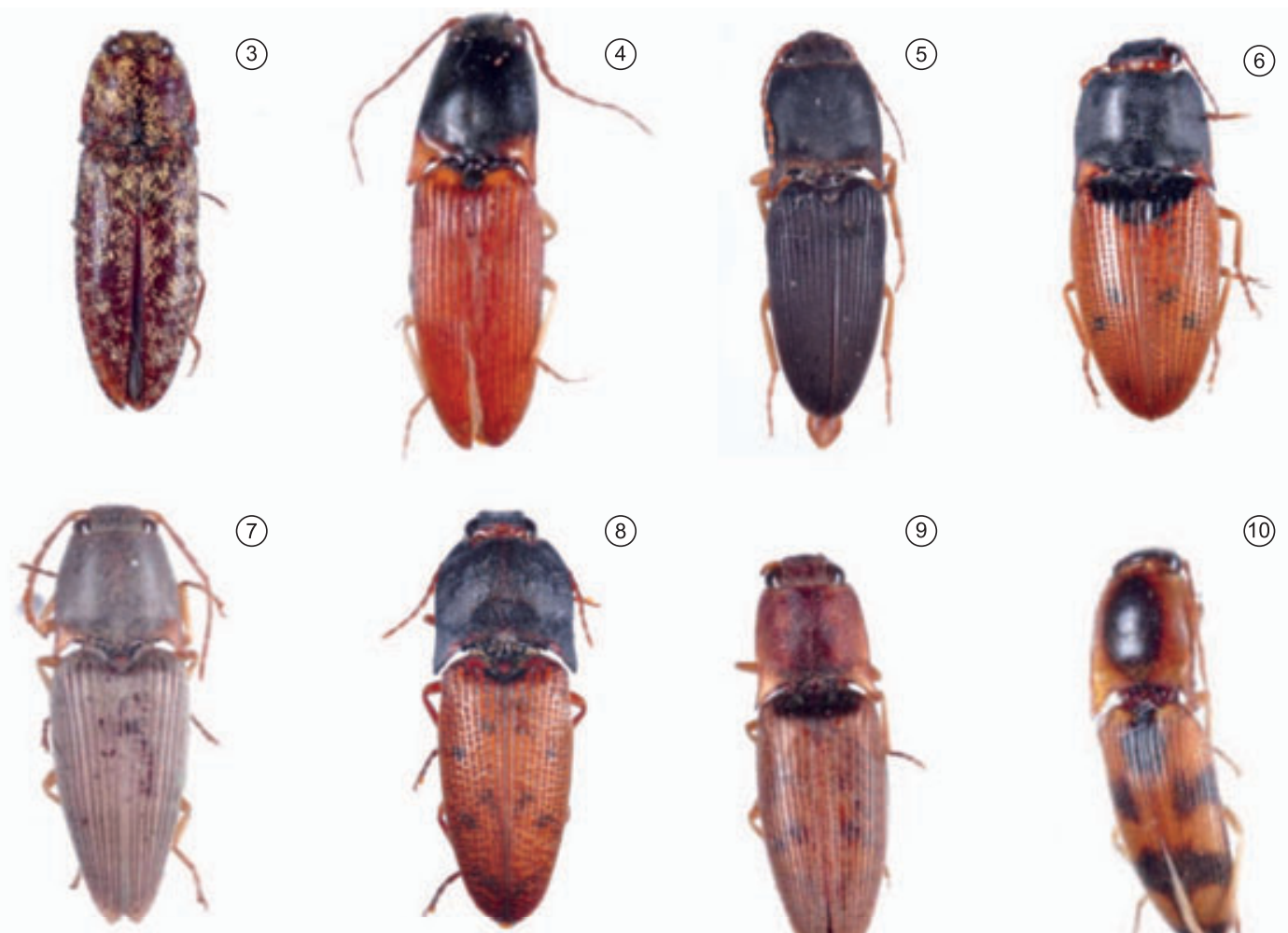

(9)

(10)
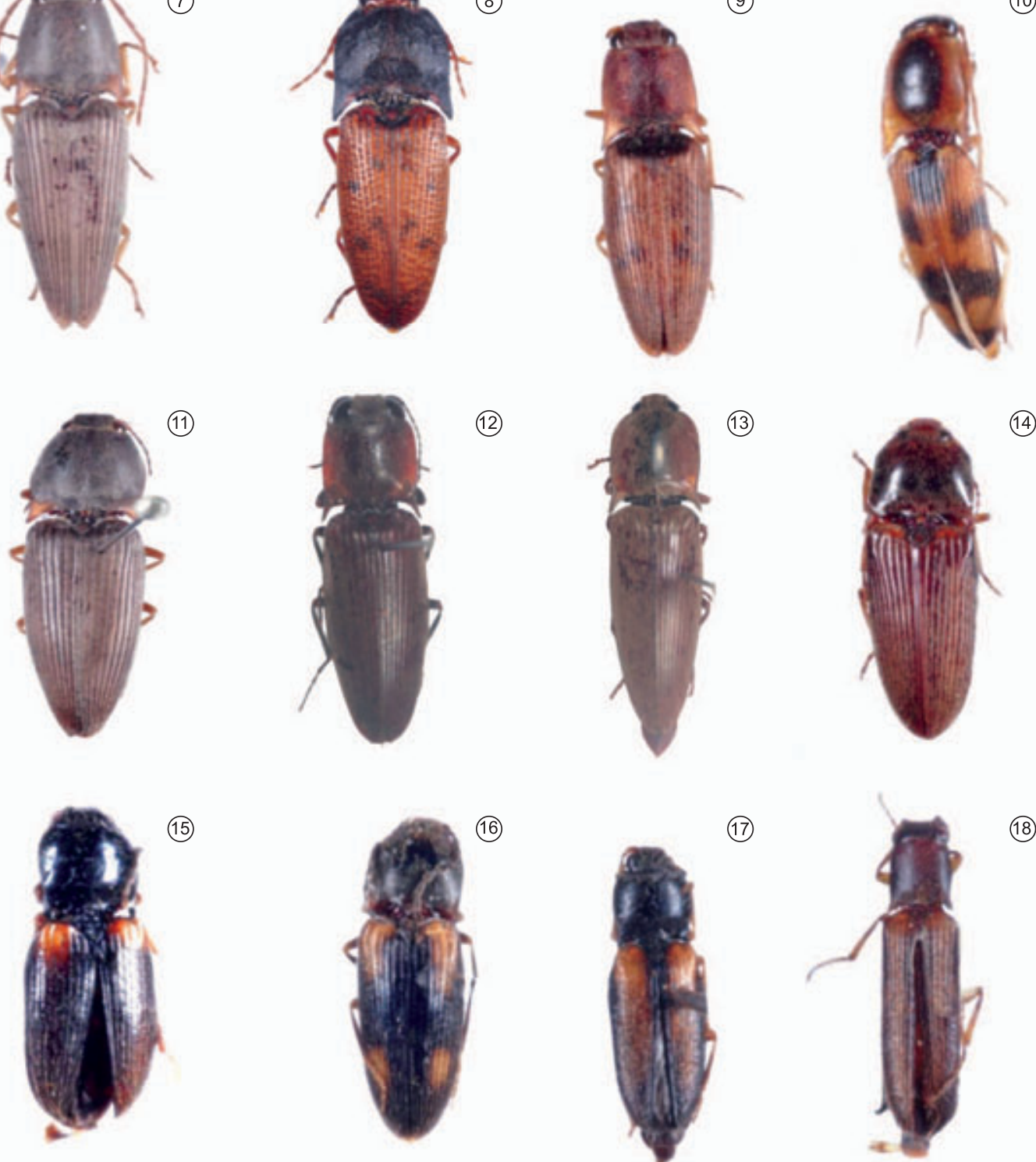

(18)

Figuras 3-18. 3) Dilobitarsus petiginosus, $10 \mathrm{~mm}$; 4) Aeolus flavipennis, $8 \mathrm{~mm}$; 5) Heteroderes laurentii, $11 \mathrm{~mm}$; 6) Conoderus inquinatus, $19 \mathrm{~mm}$; 7) Conoderus leucophaetus, $13 \mathrm{~mm}$; 8) Conoderus stigmosus, $16 \mathrm{~mm}$; 9) Conoderus sp., $11 \mathrm{~mm}$; 10) Conoderus sp.2, 6 mm; 11) Conoderus sp.3, 21 mm; 12) Opselater pyrophanus, $28 \mathrm{~mm}$; 13) Pyrearinus brevicollis, $17 \mathrm{~mm}$; 14) Esthesopus castaneus, $9 \mathrm{~mm}$; 15) Esthesopus delinitor, $4 \mathrm{~mm}$; 16) Esthesopus placidus, $5 \mathrm{~mm}$; 17) Esthesopus sp.1, $6 \mathrm{~mm}$; 18) Cylindroderus vittatus, $7 \mathrm{~mm}$.

Figures 3-18. 3) Dilobitarsus petiginosus, $10 \mathrm{~mm}$; 4) Aeolus flavipennis, $8 \mathrm{~mm}$; 5) Heteroderes laurentii, $11 \mathrm{~mm}$; 6) Conoderus inquinatus, 19 mm; 7) Conoderus leucophaetus, $13 \mathrm{~mm}$; 8) Conoderus stigmosus, $16 \mathrm{~mm}$; 9) Conoderus sp., $11 \mathrm{~mm}$; 10) Conoderus sp.2, 6 mm; 11) Conoderus sp.3, $21 \mathrm{~mm}$; 12) Opselater pyrophanus, $28 \mathrm{~mm}$; 13) Pyrearinus brevicollis, $17 \mathrm{~mm}$; 14) Esthesopus castaneus, $9 \mathrm{~mm}$; 15) Esthesopus delinitor, $4 \mathrm{~mm}$; 16) Esthesopus placidus, $5 \mathrm{~mm}$; 17) Esthesopus sp.1, $6 \mathrm{~mm}$; 18) Cylindroderus vittatus, $7 \mathrm{~mm}$. 

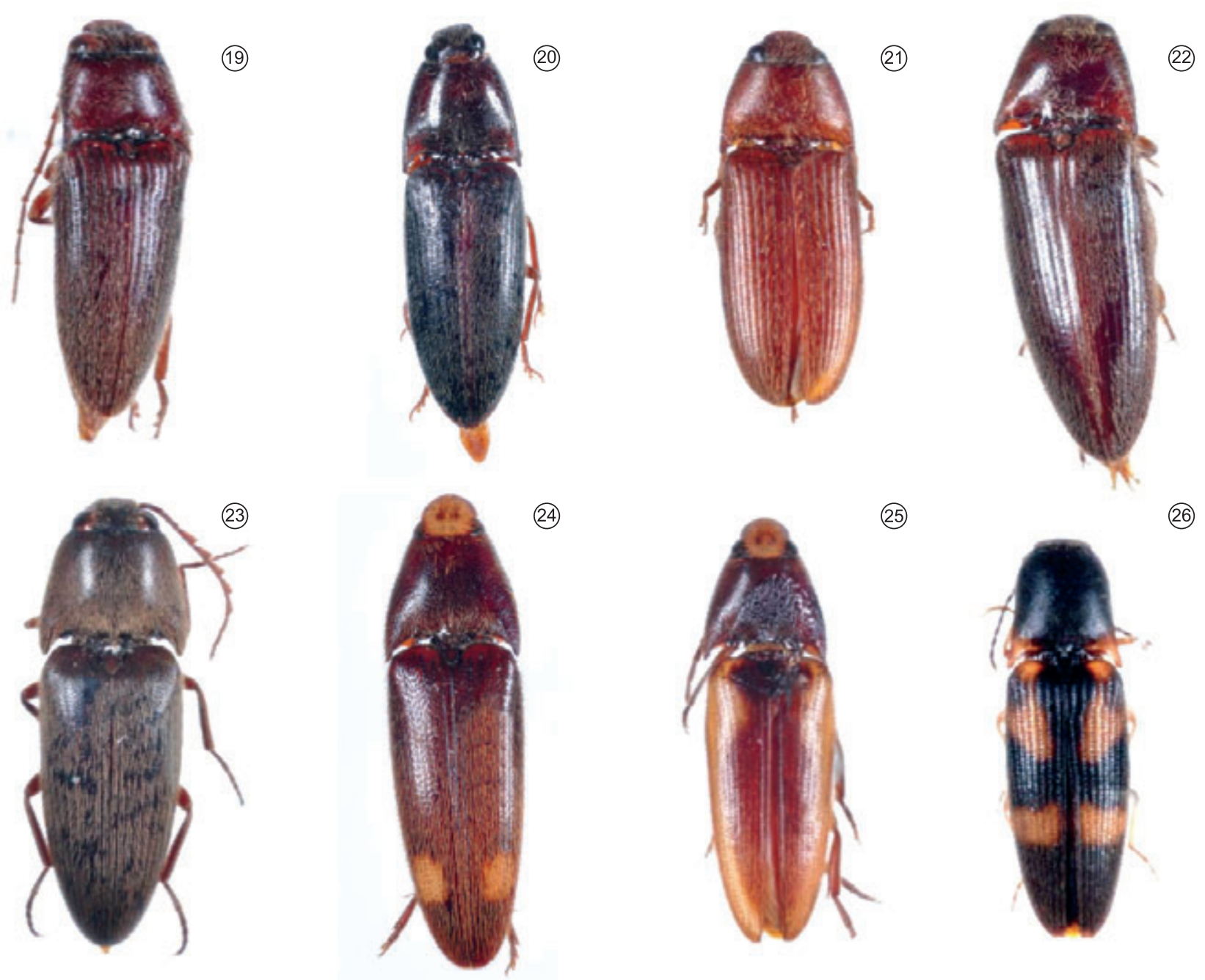

(26)
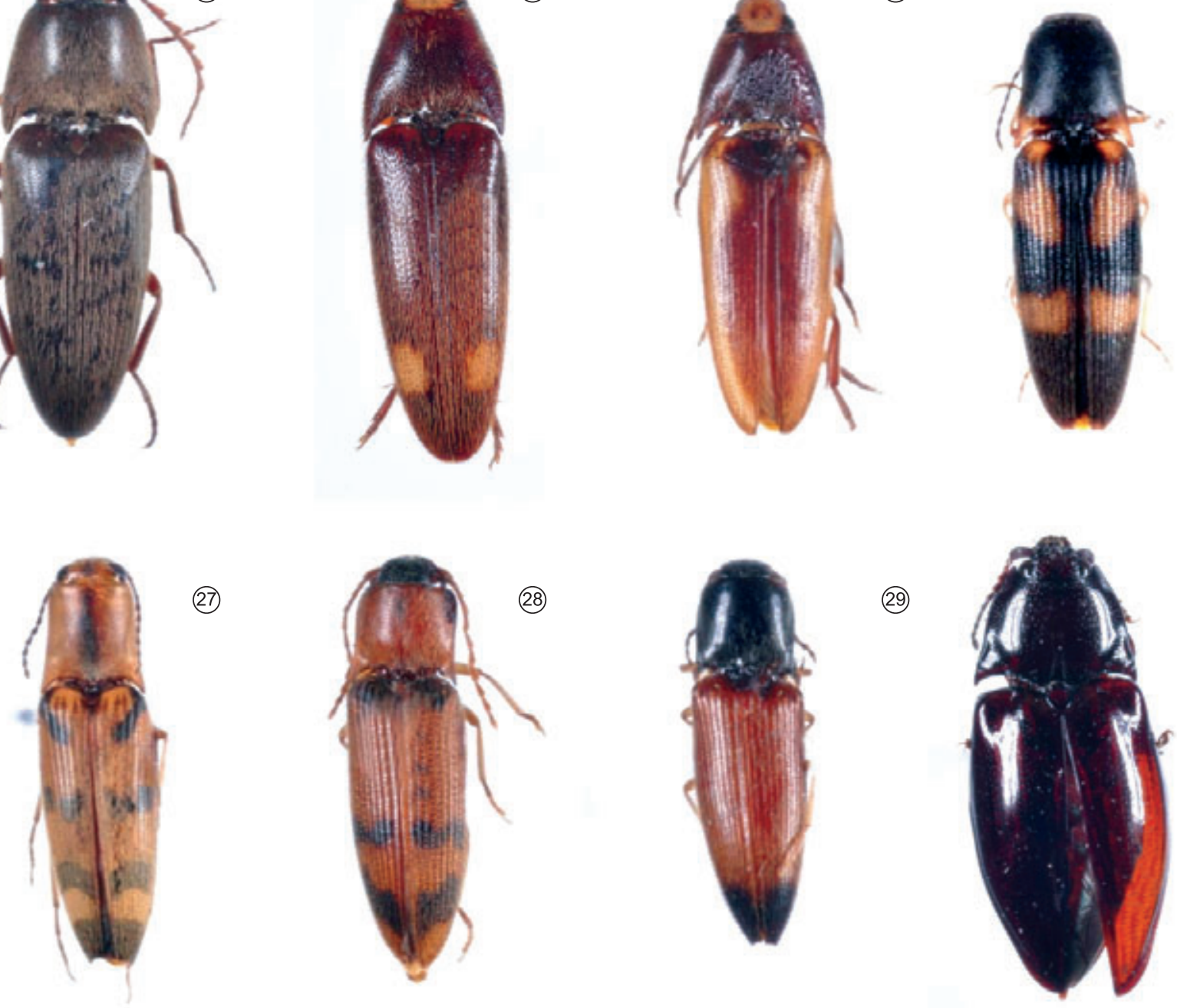

(29)

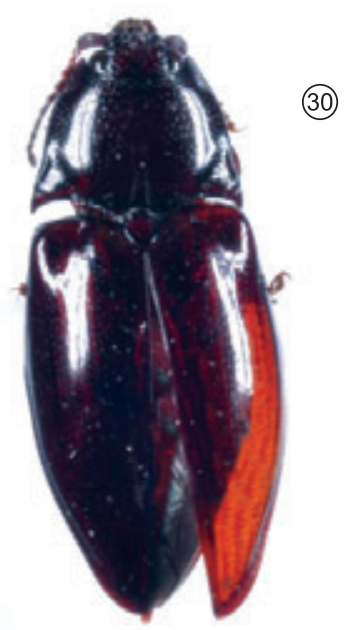

Figuras 19-30. 19) Crepidius sp.1, $11 \mathrm{~mm}$; 20) Dipropus sp.1, $14 \mathrm{~mm}$; 21) Heterocrepidius sp.1, $9 \mathrm{~mm}$; 22) Spilus sp.1, 14 mm; 23) Probothrium velutinum, $15 \mathrm{~mm}$; 24) Physorhinus distigma, $13 \mathrm{~mm}$; 25) Physorhinus xanthocephalus, $11 \mathrm{~mm}$; 26) Cosmesus sp.1, $8 \mathrm{~mm}$; 27) Cosmesus sp.2, 11 mm; 28) Pomachilini, $9 \mathrm{~mm}$; 29) Pomachilius terminatus, $7 \mathrm{~mm}$; 30) Lissomus punctulatus, $10 \mathrm{~mm}$.

Figures 19-30. 19) Crepidius sp.1, $11 \mathrm{~mm}$; 20) Dipropus sp.1, $14 \mathrm{~mm}$; 21) Heterocrepidius sp.1, $9 \mathrm{~mm}$; 22) Spilus sp.1, $14 \mathrm{~mm}$; 23 ) Probothrium velutinum, $15 \mathrm{~mm}$; 24) Physorhinus distigma, $13 \mathrm{~mm}$; 25) Physorhinus xanthocephalus, $11 \mathrm{~mm}$; 26) Cosmesus sp.1, $8 \mathrm{~mm}$; 27) Cosmesus sp.2, 11 mm; 28) Pomachilini, $9 \mathrm{~mm}$; 29) Pomachilius terminatus, $7 \mathrm{~mm}$; 30) Lissomus punctulatus, $10 \mathrm{~mm}$. 
Material Examinado: BRASIL, Rio de Janeiro: Angra dos Reis (Ilha Grande, Vila Dois Rios) Trilha da Parnaioca, 1) 26.I.2008, 1) 27.I.2008, 2) 18.VII.2008, 1) 19.VII.2008, 1) 20.VII.2008, 5, 05.IX.2008, 5, 06.IX.2008, 4, 10.X.2008, 1) 12.X.2008, 1) 13.XII.2008, Projeto Coleoptera col. ( DZRJ, MZSP).

13-Esthesopus delinitor Candèze, 1860 (Figura 15)

Distribuição(Candéze, 1860): Brasil (Rio de Janeiro)

Material Examinado: BRASIL, Rio de Janeiro: Angra

dos Reis (Ilha Grande, Vila Dois Rios) Trilha da Parnaioca, 2) 10.X.2008, 1) 11.X.2008, 1) 12.X.2008, Projeto Coleoptera col. (DZRJ, MZSP).

14-Esthesopus placidus (Eschscholtz, 1829) (Figura 16)

Distribuição (Candèze, 1860): Brasil (Rio de Janeiro).

Material Examinado: BRASIL, Rio de Janeiro: Angra dos Reis (Ilha Grande, Vila Dois Rios) Trilha do Cavalinho, 1) 12.X.2008; Trilha do Caxadaço, 1) 06.IX.2008, 2) 07. IX.2008, 1) 11.X.2008; Trilha da Parnaioca, 5, 10.X.2008, 2) 11.X.2008, 1) 12.X.2008, Projeto Coleoptera col. ( DZRJ, MZSP).

\section{5-Esthesopus sp.1 (Figura 17)}

Material Examinado: BRASIL, Rio de Janeiro: Angra dos Reis (Ilha Grande, Vila Dois Rios) Trilha da Parnaioca, 2) 10.X.2008, 1) 12.X.2008 Projeto Coleoptera col. (DZRJ, MZSP).

\section{SUBFAMÍLIA DENTICOLLINAE}

16-Cylindroderus vittatus Candèze, 1863 (Figura 18)

Distribuição (Blackwelder 1944): Brasil e Uruguai.

Material Examinado: BRASIL, Rio de Janeiro: Angra dos Reis (Ilha Grande, Vila Dois Rios) Trilha Caxadaço, 1) 07. IX.2008, 1) 11.X.2008 Trilha da Parnaioca, 1) 10.X.2008, Projeto Coleoptera col. (DZRJ, MZSP).

\section{SUBFAMÍLIA ELATERINAE}

\section{TRIBO Dicrepidiini}

\section{7-Crepidius sp.1 (Figura 19)}

Material examinado: BRASIL, Rio de Janeiro: Angra dos Reis (Ilha Grande, Vila Dois Rios) Trilha da Parnaioca, 1) 12.X.2008, 1) 13.XII.2008, Projeto Coleoptera col. (DZRJ, MZSP).

18-Dipropus sp.1 (Figura 20)

Material Examinado: BRASIL, Rio de Janeiro: Angra dos Reis (Ilha Grande, Vila Dois Rios) Trilha da Jararaca, 1) 27.I.2008; Trilha Parnaioca, 1) 17.I.2008, Projeto Coleoptera col. (DZRJ, MZSP).

\section{9-Heterocrepidius sp.1 (Figura 21)}

Material Examinado: BRASIL, Rio de Janeiro: Angra dos Reis (Ilha Grande, Vila Dois Rios) Trilha do Caxadaço, 2) 26.I.2008, 1) 16.III.2008; Trilha da Jararaca, 2) 12.XII.2008; Trilha da Parnaioca, 1) 27.I.2008, 1) 13.XII.2008. Projeto Coleoptera col. (DZRJ, MZSP).

20-Spilus sp.1 (Figura 22)

Material Examinado: BRASIL, Rio de Janeiro: Angra dos Reis (Ilha Grande, Vila Dois Rios) Trilha do Caxadaço, 3, 11.X.2008; Trilha da Parnaioca, 5, 10.X.2008, 1) 11.X.2010, 1) 13.XII.2008, Projeto Coleoptera col. (DZRJ, MZSP).
TRIBO Elaterini

21-Probothrium velutinum (Germar, 1844) (Figura 23)

Distribuição (Blackwelder 1944): Brasil.

Material Examinado: BRASIL, Rio de Janeiro: Angra dos Reis (Ilha Grande, Vila Dois Rios) Trilha do Caxadaço, 1) 25.I.2008; Trilha da Parnaioca, 1) 15.III.2008, 1) 12.X.2008; 9, 13.XII.2008, Projeto Coleoptera col. (DZRJ, MZSP).

TRIBO Physorhinini Candèze

22-Physorhinus distigma Candèze, 1859 (Figura 24)

Distribuição (Blackwelder 1944): Brasil.: México, Guatemala, Nicarágua, Panamá, Colômbia, Paraguai e Brasil.

Material Examinado: BRASIL, Rio de Janeiro: Angra dos Reis (Ilha Grande, Vila Dois Rios) . Trilha da Parnaioca, 1) 20. VII.2008, 1) 05.IX.2008, Projeto Coleoptera col. (DZRJ, MZSP)

23-Physorhinus xanthocephalus Germar, 1840 (Figura 25)

Distribuição (Blackwelder 1944): Brasil.

Material Examinado: BRASIL, Rio de Janeiro: Angra dos Reis (Ilha Grande, Vila Dois Rios) Trilha da Parnaioca, 1) 10.X.2008, Projeto Coleoptera col. (DZRJ).

TRIBO Pomachilini Candèze

24-Cosmesus sp.1 (Figura 26)

Material Examinado: BRASIL, Rio de Janeiro: Angra dos Reis (Ilha Grande, Vila Dois Rios) Armadilha Luminosa, 1) 12.X.2008; Trilha da Caxadaço, 6, 06.IX.2008, 6, 07.IX.2008, 4, 11.X.2008; Trilha da Parnaioca, 1) 11.X.2008, 1) 13.XII.2008, Projeto Coleoptera col. (DZRJ, MZSP).

25-Cosmesus sp.2 (Figura 27)

Material Examinado: BRASIL, Rio de Janeiro: Angra dos Reis (Ilha Grande, Vila Dois Rios) Trilha do Caxadaço, 1) 11.X.2008, Trilha da Paranoica, 1) 05.IX.2008, 1) 06. IX.2008, Projeto Coleoptera col. (DZRJ, MZSP).

26-Pomachilini (Figura 28)

Material Examinado: BRASIL, Rio de Janeiro: Angra dos Reis (Ilha Grande, Vila Dois Rios) Trilha da Parnaioca, 2) 05. IX.2008, 2) 06.IX.2008, 3, 10.VIII.2008, Trilha do Caxadaço, 3, 20.VII.2008, 2) 10.X.2008. Projeto Coleoptera col. (DZRJ, MZSP).

27-Pomachilius terminatus Candèze, 1860 (Figura 29)

Distribuição (Blackwelder 1944): Brasil.

Material Examinado: BRASIL, Rio de Janeiro: Angra dos Reis (Ilha Grande, Vila Dois Rios) Trilha do Caxadaço, 1) 26.I.2008, 5, 11.X.2008; Trilha Parnaioca, 2) 05.IX.2008, 2) 06.IX.2008, 34, 10.X.2008, 9, 11.X.2008, 4, 12.X.2008. Projeto Coleoptera col. (DZRJ, MZSP).

\section{SUBFAMÍLIA LISSOMINAE}

28-Lissomus punctulatus (Dalman, 1824) (Figura 30)

Distribuição (Johnson 2011): Guatemala, Nicarágua, Costa Rica, Panamá, Colômbia, Brasil e Argentina.

Material Examinado: BRASIL, Rio de Janeiro: Angra dos Reis (Ilha Grande, Vila Dois Rios, Trilha da Parnaioca), 1) 13. XII.2008, Projeto Coleoptera col. (DZRJ). 


\section{Agradecimentos}

Ao Centro de Estudos Ambientais e Desenvolvimento Sustentável (CEADS) da Universidade do Estado do Rio de Janeiro pelo apoio logístico e alojamento. Ao IBAMA e à Fundação Instituto Estadual de Florestas (IEF.RJ e INEA) pelas autorizações para pesquisa científica e licenças de coletas (respectivamente, processo $\mathrm{n}^{\circ} 10710$ 1; 10663 e 002/2008). À FAPERJ pelo suporte financeiro (Processos E-26/171.281/2006, E-26/170.502/2007, E-26/100.614/2009, E-26/101.476/2010). À Simone Policena Rosa (MZSP) pelas discussões e ajuda na identificação do material e Daniela M. Takiya (UFRJ) pela revisão do inglês do abstract e dois anônimos revisores.

\section{Referências Bibliográficas}

BLACKWELDER, R.E. 1944. Checklist of Coleopterous insects of Mexico, Central America, the West Indies and South America. Bull. U. S. Natl. Mus. 185(2):280-303.

CANDÈZE, E. 1859. Monographie des Élatérides. Tome deuxième. Mém. Soc. Roy. Sci. Liège 14:1-543.

CANDÈZE, M. E. 1860. Monographie des Élatérides. Mém. Soc. Roy. Sci. Liège, 3:1-511.

COSTA, C. 1979. Novas Espécies do Gênero Hypsiophthalmus Latreille, 1834 e Revalidação de Pyrearinus brevicollis (Eschscholtz, 1829), comb.n. (Coleoptera, Elateridae, Pyrophorinae). Pap. Avulsos Zool. 32:261-276.

COSTA, C. 2000. Estado de conocimiento de los Coleoptera neotropicales. In Hacia un Proyecto CYTED para el inventario y estimacion de la diversidad entomológica en Iberoamérica: PrIBES 2000 (F. Martín-Piera, J.J. Morrone \& A. Melic, eds). Monografías Tercer Milenio, SEA, Zaragoza, v.1, p.99-114.
COSTA, C., LAWRENCE, J.F. \& ROSA, S.P. 2010. Elateridae Leach, 1815. In Handbook of Zoology. Arthropoda: Insecta. Coleoptera. Beetles. Morphology and systematics (Elateroidea, Bostrichiformia, Cucujiformia partim) (R.A.B. Leschen, R.G. Beutel \& J.F. Lawrence, eds). De Gruyter, Berlin, v.2, p.75-103.

JOHNSON, P.J. 2002. Elateridae Leach 1815. In American Beetles. Polyphaga: Scarabaeoidea through Curculionoidea (R.H. Arnett Junior, M.C. Thomas, P.E. Skelley \& J.H. Frank, eds.). CRC Press, Gainesville, v.2, p.160-173.

JOHNSON, P.J. 2011. Costa Rican Beetle Websites. In South Dakota State University. http://www.sdstate.edu/ps/Severin-McDaniel/project-elater/ caribbean-atlantic-southernnorthamerica/costa-rica/elateridae/lissomus. cfm (último acesso em 07/02/2011).

LAWRENCE, J.F. \& NEWTON, A.F. 1995. Families and subfamilies of Coleoptera (with selected genera, notes, references and data on family-group names). In: Biology, Phylogeny, and Classification of Coleoptera (J. Pakaluk \& S.A. Slipinski, eds.). Museum I Instytut Zoologii PAN, Warszawa, p.7791006. Papers Celebrating the 80nd Birthday of Roy A. Crowson.

LIMA, A.M.C. 1953. Insetos do Brasil. Coleopteros. Escola Nacional de Agronomia, Rio de Janeiro, tomo 8.

MITTERMEIER, R.A., GIL, P.R., HOFFMANN, M., PILGRIM, J., BROOKS, T., MITTERMEIER, C.G., LAMOUREX, J., FONSECA, G.A.B. 2005. Hotspots revisited: earth's biologically richest and most endangered terrestrial ecorregions. CEMEX, Agrupación Sierra Madre, Washington.

RODRIGUES, J.M.S., MONNÉ, M.A. and MERMUDES, J.R.M. 2010. Inventory of the Cerambycida especies (Coleoptera) from Vila Dois Rios (Ilha Grande, Angra dos Reis, Rio de Janeiro, Brazil). Biota Neotrop. 10(3): http://www.biotaneotropica.org.br/v10n3/en/abstract?article+bn 00310032010(último acesso em 15/01/2011).

ROSA, S.P. 2004. Revisão do gênero Opselater Costa (Coleoptera, Elateridae, Agypninae). Rev. Bras. Entomol. 48(2):203-219. http://dx.doi. org/10.1590/S0085-56262004000200008 
